\title{
Engineering Flat Gain Tunable Raman-Parametric Hybrid L-Band Amplifier for Narrow Band Multi-Channel Terabits System
}

https://doi.org/10.1515/joc-2017-0113

Received July 07, 2017; accepted January 03, 2019

\begin{abstract}
We present a model of a Raman-parametric hybrid amplifier for flat gain amplification of narrowband Dense Wavelength Division Multiplexed (DWDM) terabits capacity system. In the proposed configuration Raman pump has been cascaded with one - pump parametric amplifier. While Raman amplifier typically uses a long length single mode fiber as a gain medium, the Fiber Optical Parametric Amplifiers (FOPA) uses a small length of Highly Non-Linear Fibers (HNLF). This allows for maximum optimization of the gain achieved using Raman as well as parametric processes. We focus on the signal degradation due to non-linear crosstalk arising because of multi-pump configuration, in particular, due to the generated idlers within the operational bandwidth. As the channel spacing is reduced, fiber non-linearities increase significantly. The dilemma to use the fewer number of high powered pumps Vs, the use of increased number of relatively low powered pumps has been investigated for DWDM system. The optimization of the pump powers has been evaluated in terms of signal degradation, gain, gain variation and OSNR. It has been demonstrated that a maximum gain of $45.38 \mathrm{~dB}$ for $96 \times 40 \mathrm{Gbps}, 25 \mathrm{GHz}$ system is attainable up to $200 \mathrm{~km}$ of transmission distance while maintaining OSNR $>18 \mathrm{~dB}$ using the proposed hybrid. No gain compensation or gain optimization technique has been used. The findings of this work establish flat gain in narrowband DWDM systems is achievable through proper optimization of pump wavelengths and their powers.
\end{abstract}

Keywords: narrow spaced DWDM, hybrid parametric amplifier, Flat Gain, pump power

\section{Introduction}

Multi-channel, Wave Length Multiplexed systems (WDM) systems are currently ruling the optical transmission

*Corresponding author: Gaganpreet Kaur, ECE Department, Thapar University, Patiala, India, E-mail: gaganpreet.kaur@thapar.edu scenario due to their high capacity and fast multiuser data transmission $[1,2]$. But as the channel spacing shrinks and data rate increases, passive losses coupled with optical nonlinearities increase manifold in DWDM systems. To maintain the minimum detectable signal to noise ratio (SNR) at the receiver, the use of optical amplifiers is necessary for these systems. FOPA with their capabilities to provide large gain, broadband amplification [3, 4] and low noise figure [5] make promising amplifiers for such systems [4, 6-10]. In [11] different hybrid configurations of Raman, EDFA(Erbium Doped Fiber Amplifier) have been evaluated for WDM systems amplification and it has been emphasized that parametric amplifiers have a much wider scope and flexibility over the existing configurations of Raman and EDFA. Jiang and Jiang in [12] demonstrated a reasonably well BER achievable for an 8-channel WDM system over distance up to $385 \mathrm{~km}$ with each fiber span of $55 \mathrm{~km}$ using FOPA cascade with Raman Effect as an effective inline amplifier. The Raman Effect as a dominant source of noise in single pump FOPAs was theoretically and experimentally investigated for determining the gain spectra of a FOPA [13]. Golovchenko et al. [14] examined the phase mismatch of parametric gain and has demonstrated that the gain depends strongly on the real part of the complex Raman susceptibility. So the contribution of Raman Effect as Raman seeded phonon noise was significant to parametric gain. Raman effect based Fiber Raman Amplifier (FRA) had in research established itself as a class of optical amplifiers capable of providing wide, tunable bandwidths in excess of $100 \mathrm{~nm}$ with gain ripple $<1.5 \mathrm{~dB}$ in WDM applications [15-17]. So, research has always been motivated to explore hybrid of FOPA and Raman amplifier as tunable broadband amplifiers [18]. The first RamanFOPA hybrid was configured in the form of Raman-assisted fiber optical parametric amplifiers (RA-FOPAs). The RAFOPA configuration offered flexibility in the selection of parametric pump powers and wavelengths coupled with the inherent advantages of FOPAs [18, 19]. RA-FOPAs provide both direct as well as indirect amplification of the signal [18]. Redyuk et al. in [20] numerically analyzed the performance of (RA-FOPA) in QPSK modulated WDM system. Performance enhancement of FOPA using Raman 
assisted pumping has been clearly established from the results offering $20 \mathrm{~dB}$ net gain in $10 \times 58 \mathrm{Gbps}$ system with $7 \mathrm{~dB}$ reductions in crosstalk over FOPA alone. Wang et al. in their work [21, 22] explored novel configurations of RAFOPA. In conventional RA-FOPAs, most energy from the Raman amplifier is trapped in the parametric pump at the output end of the amplifier. So, Wang et al. [21] proposed a hybrid fiber Raman/parametric amplifier constructed by cascading a FOPA after the RA-FOPA achieving a gain enhancement of $34 \mathrm{~dB}$ compared over conventional RAFOPA. Ummy et al. [23] demonstrated the extended flat gain of about $15 \mathrm{~dB}$ of with gain ripple of $5 \mathrm{~dB}$, using combined Raman and parametric interaction in HNLF. But Peiris et al. [24] demonstrated hybrid Raman-Optical Parametric amplifier (HROPA) in Tandem configuration for extended bandwidth, with gain $>20 \mathrm{~dB}$ and extended gain bandwidth of $170 \mathrm{~nm}$ with gain ripple $<4 \mathrm{~dB}$. The tandem configuration used the concept of single pump FOPA for two sub-bands and idler crosstalk controlled through the multiplexer filter transfer function.

In [25] improved performance with RA-FOPA for WDM system has been achieved with a net gain of $20 \mathrm{~dB}$ and gain ripple of $1.9 \mathrm{~dB}$ for $10,100 \mathrm{GHz}$ spaced DFB lasers. Use of tunable NRZ signals showed reduced susceptibility to saturation of gain in Raman-FOPA hybrid [26]. Kaur et al. [27] have demonstrated Raman-FOPA cascade for $96 \times 100$ Gbps system with $25 \mathrm{GHz}$ spacing to give a gain of $14 \mathrm{~dB}$ over L-band. A hybrid amplifier of Raman cascaded with two section FOPA has been demonstrated to achieve a wide bandwidth of $220 \mathrm{~nm}$ by Kaur et al. [28]. In the proposed work RamanFOPA cascade instead of conventional Raman-assisted FOPA has been proposed because of the following reasons:
1. It allows pumps of Raman and FOPA to be independently tuned in complementary regions. Raman amplification requires longer interaction lengths while parametric amplifiers use short length HNLFs.

2. Raman Fiber can be utilized for amplification as well as dispersion compensation allowing implementation of Raman amplifier in distributed or discrete configuration.

3. The amplified output of Raman amplifier serves as input to FOPA. This increased signal power at its input helps achieve better FWM efficiency responsible for parametric amplification.

\section{Experimental set up}

Set up comprises of a DWDM system uniformly spaced 96 channels in L-band from $187 \mathrm{THz}$ to $189.375 \mathrm{THz}$ as shown in Figure 1. The RZ modulated signals at $40 \mathrm{Gbps}$ are spaced at $25 \mathrm{GHz}$. In the given model, signal traverses $100 \mathrm{~km}$ of transmission dispersion compensated using Dispersion Compensation Fiber (DCF). The proposed system used $12 \mathrm{~km}$ of FRA while $200 \mathrm{~m}$ HNLF is used in FOPA. The HNLF parameters used are as specified in Table 1. The aim of this investigation of the proposed hybrid is to achieve flat gain amplification in narrow spaced multi-channel systems without using any gain equalization/compensation technique. This is achieved via careful optimization of the number of pumps, their wavelengths and their powers both for Raman and FOPA fiber amplifiers in the cascade.

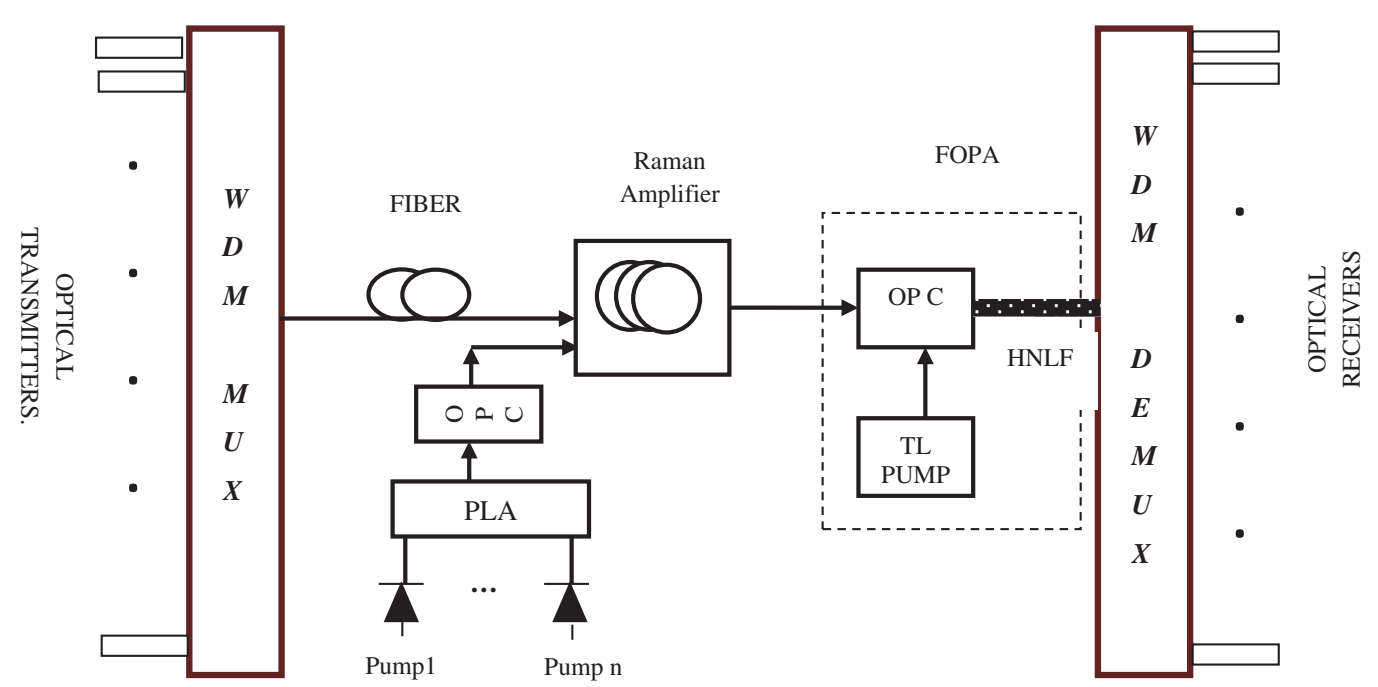

Figure 1: Schematic for Experimental setup. FRA-Fiber Raman Amplifier, OPC-Optical Power Coupler, PLA-Pump laser Array, TL-tunable laser. 
Table 1: HNLF parameters used.

\begin{tabular}{lr}
\hline Parameter & Value \\
\hline HNLF dispersion slope & $0.02 \mathrm{ps} / \mathrm{nm}^{2} \mathrm{~km}$ \\
HNLF coefficient & $17.83(\mathrm{Wkm})^{-1}$ \\
HNLF Length & $200 \mathrm{~m}$ \\
HNLF attenuation & $0.8 \mathrm{~dB} / \mathrm{km}$ \\
\hline
\end{tabular}

In the first phase of our investigation, the experimental set up of Figure 1 uses a Raman amplifier with three pumps from $1480 \mathrm{~nm}$ to $1505 \mathrm{~nm}$ and one parametric pump at $189.315 \mathrm{THz}$. The wavelengths of Raman and parametric pumps are so tuned that they amplify complimentary sub-bands of bandwidth under consideration. Raman-FOPA cascade serves as an inline amplifier in our set up. The input signal after traversing transmission fiber of $100 \mathrm{~km}$ is first launched into the Raman amplifier. $12 \mathrm{~km}$ of SMF has been used in Raman amplifier with back-propagating pumps. Unlike Raman assisted FOPA using the same piece of HNLF [22], separate SMF has been used in Raman amplifier. This helps achieve a higher gain in proposed cascade as Raman amplification is based on longer interaction lengths whereas parametric amplification uses short length HNLF.

Raman amplified signal is then coupled to Continuous wave (CW) laser source serving as the pump for parametric amplification at $187.315 \mathrm{THz}$.

The Raman gain has been modeled as given by [29] as:

$$
G_{\text {Raman }}=10 \log _{10}\left(\exp \left(g_{R} P \frac{L_{e f f}}{A_{e f f}}\right)-\alpha L\right)
$$

where $g_{R}$ is Raman gain coefficient, $P$ is pump power, $\mathrm{Le}_{\mathrm{ff}}$ is effective fiber length in Raman amplifier, $A_{\text {eff }}$ is effective fiber area and $\alpha$ is fiber attenuation.

The gain of FOPA for the dual pump is given as [30]:

$$
G_{F O P A}=1+\left\{2 \gamma \frac{\sqrt{P_{1} P_{2}}}{g} \sinh (g L)\right\}^{2}
$$

where, $\gamma$ is non-linear coefficient, $P_{1}, P_{2}$ are pump powers, $\mathrm{L}$ is the length of fiber and $\mathrm{g}$ is the parametric gain coefficient

$$
g=\sqrt{4 \gamma^{2} P 1 P 2-\left(\frac{k+\delta k}{2}\right)^{2}}
$$

where $\mathrm{k}$ is phase mismatch and given by:

$$
k=\beta_{2} \omega_{c}\left(\Delta \omega_{s}^{2}-\Delta \omega_{P}^{2}\right)+\frac{1}{12} \beta_{4} \omega_{c}\left(\Delta \omega_{s}^{4}-\Delta \omega_{P}^{4}\right)+\gamma\left(P_{1}+P_{2}\right)
$$

$\omega_{c}=\frac{1}{2}\left(\omega_{1}+\omega_{2}\right)$ for 2 pumps at $\mathrm{P}_{1}$ and $\mathrm{P}_{2}$. For single pump FOPA $\mathrm{P}_{1}=\mathrm{P}_{2}=\mathrm{P}$.

The gain expected of the used novel multi-pump Raman-FOPA cascade hybrid is:

$$
\mathrm{G}_{\text {Raman }} \text {-FOPACascade }=\mathrm{G}_{\text {Raman_amp }}+\mathrm{G}_{\text {FOPA_1pump }}
$$

where $G_{\text {Raman_amp }}$ is the individual gain of the Raman amplifier and $\mathrm{G}_{\mathrm{FOPA} \_ \text {1pump }}$ is the individual gain of a single parametric amplifier

In the proposed model, the state of polarization of the input and pumps has been assumed to be aligned, which may not always be true in practical systems.

\section{Results and discussion}

\subsection{Use multi-pump Raman high power pumps and optimizing FOPA pump frequency}

The proposed configuration uses three Raman pumps equally spaced at $1485 \mathrm{~nm}, 1495 \mathrm{~nm}$ and $1505 \mathrm{~nm}$ with respective pump powers as $650 \mathrm{~mW}, 1000 \mathrm{~mW}$ and 950 $\mathrm{mW}$ to provide gain in L-band from $1583 \mathrm{~nm}$ to $1603 \mathrm{~nm}$. The $100 \mathrm{~km}$ fiber span has been dispersion compensated using $19.56 \mathrm{~km}$ of DCF with $\mathrm{D}=-90 \mathrm{ps} / \mathrm{nm} / \mathrm{km}$ and $\mathrm{S}=0.112 \mathrm{ps} / \mathrm{nm}^{2} / \mathrm{km}$. A parametric pump is placed at 189.315 $\mathrm{THz}$ with $23 \mathrm{dBm}$ power.

Results in Figure 2(a) show remarkable gain flatness of $5.15 \mathrm{~dB}$ with the highest gain of $32.63 \mathrm{~dB}$ and a minimum gain of $27.48 \mathrm{~dB}$ without any gain compensation technique. The achievement of high gain is attributed primarily due to use of high pump powers of the order of $650 \mathrm{~mW}$. As seen from the Raman gain profile indicated by blue curve gain falls at higher frequencies (shorter wavelengths). To compensate for this fall in gain we use a high power parametric pump at higher frequency region thereby compensating for the fall in Raman gain. Resulting gain due to the combined effect of Raman and FOPA is a flat gain indicated by the red curve in Figure 2(a). Figure 2(b) shows the variation in FOPA gain due to the change in relative pump frequency. 

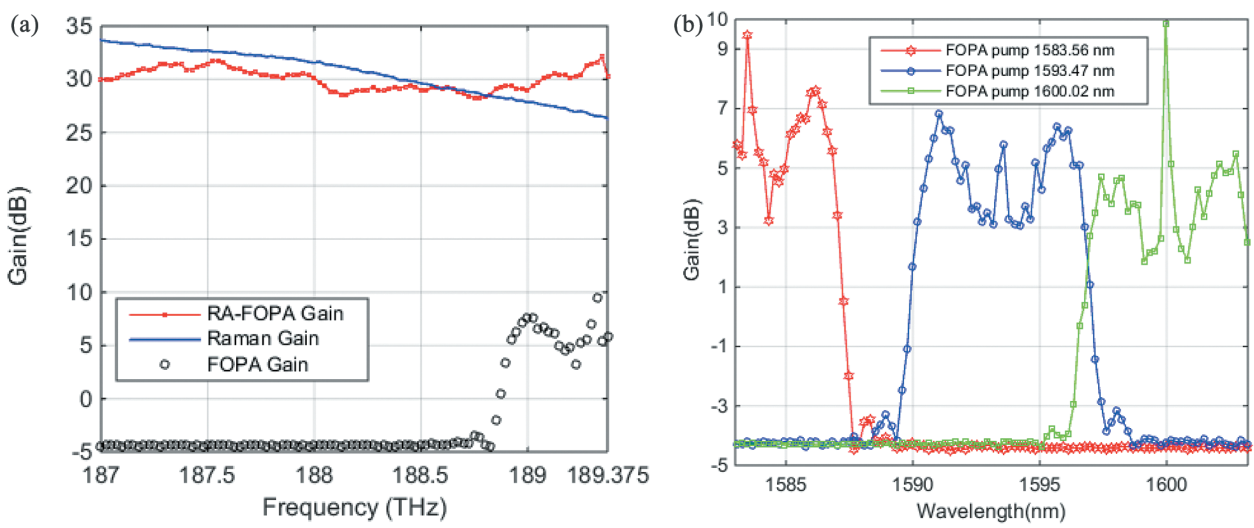

Figure 2: (a) Gain Profile of Raman-FOPA hybrid, Raman and FOPA in proposed set up. (b) Parametric gain with varying relative pump power wavelength.

More is the difference between relative pump frequency and reference wavelength, a more asymmetric and peaked gain profile is achieved In the current work as gain flatness is the objective peaked curves are not preferred and hence pump wavelength of $1583.56 \mathrm{~nm}$ (189.315THz) gives optimized results.

\subsection{Optimizing number of Raman pumps between high power pumps and increased number of low power pumps}

In this phase of the investigation, the attempt has been made to identify between the use of fewer high powered pumps or use of increased number of Raman pumps with low power. The motivation for this investigation comes from research results indicating low gain with the use of pump powers higher than $650 \mathrm{~mW}$ [17]. We investigate three different configurations of Raman amplifier based on its average power model- using three pumps with high powers of more than $650 \mathrm{~mW}$, seven Raman pumps with mid-range powers varying between $200 \mathrm{~mW}$ and 350 $\mathrm{mW}$ and a configuration using 15 Raman pumps with the uniform low power of $100 \mathrm{~mW}$. The gain profile for proposed Raman-FOPA cascade employing three different Raman pump configurations has been shown in Figure 3(a). The black curve indication Raman-FOPA gain when employing 15 Raman pumps of the low power of $100 \mathrm{~mW}$ shows a very poor gain of less than $5 \mathrm{~dB}$ with a sudden increase in gain up to $14.89 \mathrm{~dB}$ at higher frequencies. The black curve in Figure $3(\mathrm{~b})$ helps us identify the gain of less than $5 \mathrm{~dB}$ is contributed by Raman amplifier while the sudden increase in gain in contributed due to high pump FOPA tuned at higher frequencies. This clearly shows the gain profile of Raman-FOPA cascade dominated by FOPA. In Figure 3(a) the blue curve shows improved gain and gain flatness when seven Raman pumps are used at mid-range powers of less than $350 \mathrm{~mW}$. The third and most important observation comes with reference to our proposed RamanFOPA cascade that to maintain the gain flatness between two band extremities Raman pump power should be of higher order than the FOPA pump. This is because in Raman amplifier dominant noise sources are contributed primarily by Double Rayleigh Scattering (DRS) and Amplified Spontaneous Noise (ASE) [26]. In the multipump configuration, the dominant source of noise is pump-pump interaction [31]. Since ASE manifests itself as beat signal [17] produced on interaction with the copropagating signal it is accounted for in idler signal generated due to FWM in proposed Raman-FOPA cascade. These idlers falling on original signals result in a sharp fall in the gain of the transmitted signals. DRS, on the other hand, is believed to limit the gain per stage when used over multiple stages in long-haul communications. This is well observed from OSNR variation of two-stage amplification OSNR profiles shown in Figure 4.

Apart from noise sources in Raman amplifier, FOPA suffers crosstalk both from transmission fiber nonlinearities as well as nonlinear effects of the parametric amplifier itself. As the number of channels increases in WDM system the FOPA crosstalk dominates over transmission channel non-linear effects [9]. The dominant nonlinear effects of FOPA are Four-wave Mixing (FWM) and Cross Gain modulation (XGM). This nonlinear crosstalk is coupled with ASE and Raman scattering noise which coincides with the bandwidth of parametric amplification.

Though the parametric pump used in the proposed model is not EDFA amplified, ASE due to parametric pump is ignored but the amplified quantum noise [32] leads to idler signal variations at output thereby affecting 

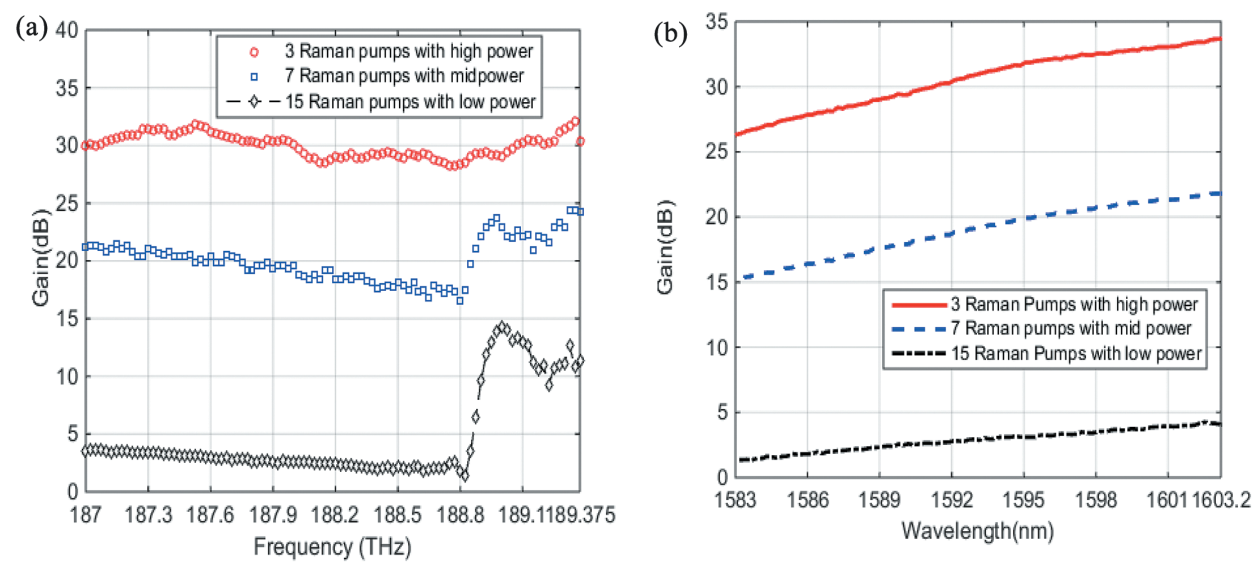

Figure 3: (a) Gain variations of the proposed hybrid using different Raman pumps. (b) Gain variations of the Raman amplifier with different pumps.
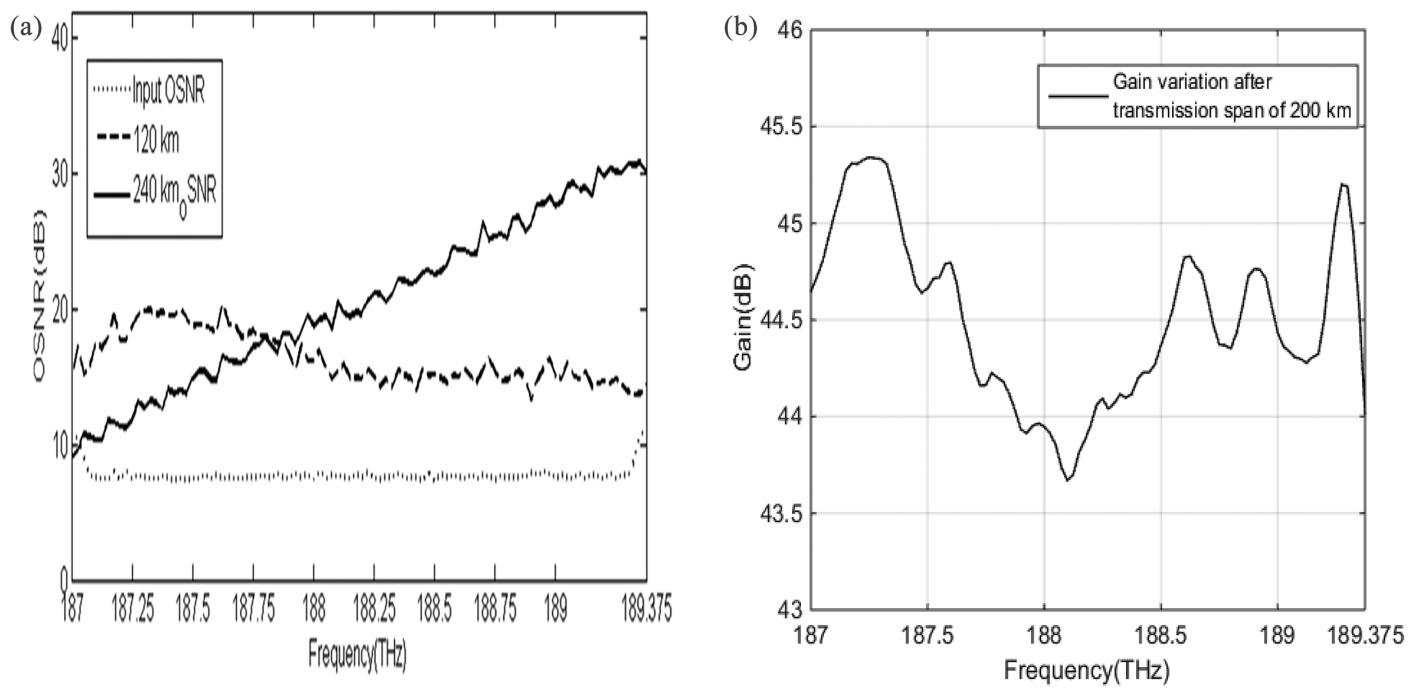

Figure 4: OSNR variation for Raman-FOPA cascade amplifier employing 7 Raman pumps of powers less than $350 \mathrm{~mW}$.

the parametric gain. The parametric pump alone can provide high gain over wide bandwidth but with the increase in the number of channels single pump FOPA is not effective to provide significant amplification Wang et al. [21] derived a theoretical model for RA-FOPA using multiple Raman pump assisted FOPAs. Flat gain over 40 $\mathrm{nm}$ bandwidth is demonstrated with gain enhancement of $15 \mathrm{~dB}$ over conventional FOPA. This makes RamanFOPA cascade attractive for terabits multichannel systems. From the analysis of noise sources in Raman and parametric amplifiers above it may be established under given set of assumption for the state of polarization, and absence of EDFA pumped source, use of multiple Raman pumps at high powers is the dominant cause of increased quantum and beat signal noise in the proposed set up.
After establishing three Raman pump configuration having the best gain we extend our gain results to multiple span transmission considering two spans each of $100 \mathrm{~km}$ amplified by Raman-FOPA cascade amplifier. Figure 5(a) shows the gain profile over $200 \mathrm{~km}$ transmission. The highest gain achieved is $45.38 \mathrm{~dB}$ while the minimum gain is $43.69 \mathrm{~dB}$. This reduces the gain ripple to $1.69 \mathrm{~dB}$ from the single span gain ripple of $5.15 \mathrm{~dB}$. The results obtained are very encouraging towards the use of RamanFOPA cascade amplifier for long distance transmission. To compare the seven pump configuration over multiple spans we extend transmission to $200 \mathrm{~km}$. The distribution of Raman pump powers is shown in Figure 5(b) for both the configurations whereas the gain for both configurations is shown in Figure 5(c). 

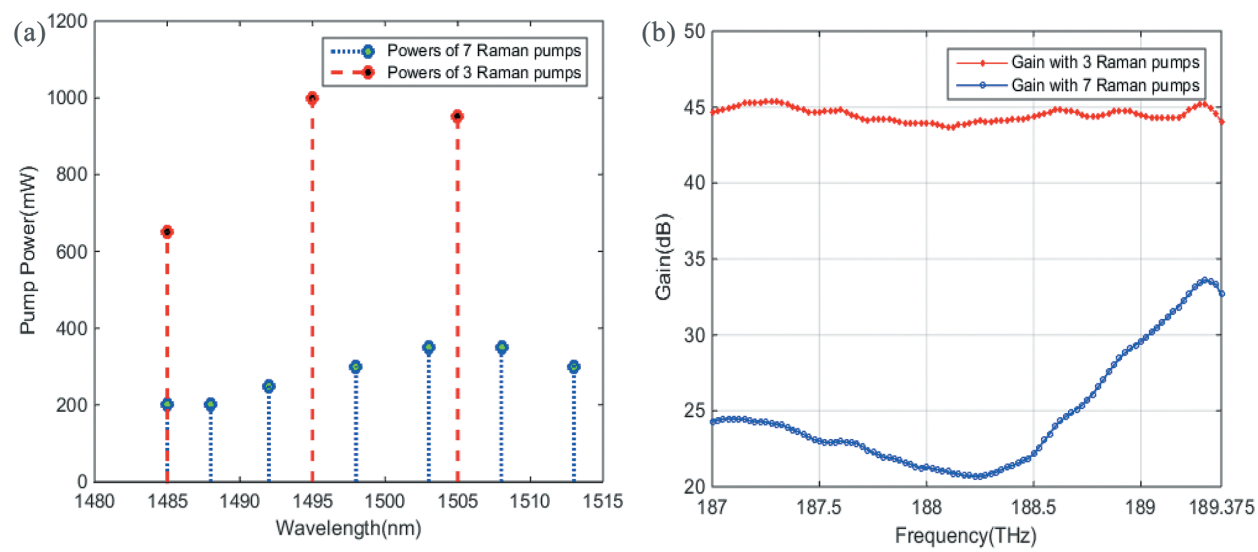

Figure 5: (a) Gain profile of Raman-FOPA cascade using 3 pump configuration after transmission of $200 \mathrm{~km}$. (b) Raman pump powers for 3 pump and 7 pump configuration. (c) Gain profile of Raman-FOPA cascade using 3 pumps (red curve) configuration and 7 pumps (blue curve) after transmission of $200 \mathrm{~km}$.

Singh and Kaler [33] in their recent work investigated and used genetic algorithm to optimize gain of Raman and EDFA hybrid optical amplifier for $25 \mathrm{GHz}, 100 \times 10 \mathrm{Gbps}$ system and attained a maximum gain of $>18 \mathrm{~dB}$ which is much lower than achieved in proposed work at $25 \mathrm{GHz}$ for $96 \times 40 \mathrm{Gbps}$ system. This is the highest gain achieved for $40 \mathrm{Gbps}$ multichannel system with narrow channel spacing of $25 \mathrm{GHz}$. No gain equalization technique has been used in the proposed design yet flat gain with a reasonably low ripple of $1.69 \mathrm{~dB}$ has been achieved over a distance of $200 \mathrm{~km}$ in L-band DWDM systems. So the proposed Raman-FOPA cascade as an inline amplifier for DWDM systems establishes its feasibility as an inline amplifier in long-haul DWDM systems with careful optimization of Raman and parametric pumps.

\section{Conclusion}

A novel Raman-FOPA cascade for L-band terabits capacity $96 \times 40$ Gbps DWDM has been proposed. The effect of Raman amplifier noise sources and parametric amplification crosstalk for multichannel DWDM has been studied. Results prove that Raman -FOPA hybrid can be tailored for any band in useful communication 1400-1650 $\mathrm{nm}$ band for flat gain without any compensation techniques. Raman- FOPA cascade gives the flexibility to tune Raman and FOPA gain in different sub-bands and hence achieve flat gain. For a multichannel system, the use of multiple pumps significantly adds to noise due to pump-pump interactions. So an attempt has been made to optimize the number of pumps and their powers to provide distributed amplification over the entire band. Single pump FOPA is then tuned with high power pump to boost the signal level in the band of frequencies where Raman gain falls. High gain can be achieved using high power pumps but leads to a significant increase in crosstalk significantly increases, making a noise comparable with that of propagating signals. Single pump FOPA has been used with high pump power whereas or non-uniform Raman pumps with powers less than $350 \mathrm{~mW}$ have been used to keep pump-pump interaction noise minimum. Distributed pumps with sufficient wavelength spacing have been used and Raman pumps are placed far from ZDWL as well pump wavelength of FOPA to minimize FOPA-Raman pump interaction crosstalk. Additionally, unlike Ramanassisted parametric amplifiers which make use of same HNLF as FOPA, the proposed cascade uses separate SMF for Raman amplification as Raman gain is proportional to the length of the fiber. This helps in Raman gain enhancement. Short lengths of HNLF of $200 \mathrm{~m}$ are used for parametric amplification. Another important finding is optimal fiber span. The optimal distance for near 100 channels transmission system is $100 \mathrm{~km}$. Increasing the fiber span beyond $100 \mathrm{~km}$ (compensated or uncompensated) reduces OSNR and signal strength drastically. This paper explores the feasibility of Raman-FOPA hybrid for terabits long-haul DWDM transmission. So far no work has been reported to achieve an average gain of more than $25 \mathrm{~dB}$ using RamanFOPA hybrid after $200 \mathrm{~km}$ of fiber transmission at $40 \mathrm{Gbps}$. The proposed hybrid can be optimized with respect to polarization sensitivity and dispersion characteristics. Dispersion characteristics of fiber used for Raman amplification can be tailored to achieve dispersion compensation as well [21]. Use 
of advanced modulation formats [34, 35] at high data rates $>80$ Gbps combined with wideband gain capabilities of parametric amplifiers $[36,37]$ can add significant results to realizing flat gain amplifiers for high capacity multichannel systems in future.

\section{References}

1. Bennett G, Wu K-T, Malik A, Roy S, Awadalla A. A review of highspeed coherent transmission technologies for long-haul DWDM transmission at $100 \mathrm{~g}$ and beyond. IEEE Commun Mag. 2014;52:102-10.

2. Yu A, Zou W, Li S, Chen J. A multi-channel multi-bit programmable photonic beam former based on cascaded DWDM. IEEE J Photonics. 2014; 6:open access.

3. Hansryd J, Andrekson P, Westlund M, Li J, Hedekvist PO. Fiberbased optical parametric amplifiers and their applications. IEEE J Sel Top Quantum Electron. 2002;8:506-20.

4. Zhang L, Yang S, Wang X, Gou D, Chen W, et al. Photonic crystal fiber based wavelength-tunable optical parametric amplifier and pico-second pulse generation. IEEE Photonics J. 2014;6: open access.

5. Voss PL, Tang R, Kumar P. Measurement of the photon statistics and the noise figure of a fiber-optic parametric amplifier. Opt Lett. 2003;28:549-51.

6. Kuo BP, Chui P, Wong KY. A comprehensive study on crosstalk suppression techniques in fiber optical parametric amplifier by modulation format. IEEE J Sel Top Quantum Electron. 2008;14:659-65.

7. Marhic ME, Kagi N, Chiang TK, Kazovsky LG. Broadband fiber optical parametric amplifiers. Opt Lett. 1996;21:573-5.

8. Marhic ME, Wong KKY, Kazovsky LG. Wideband tuning of the gain spectra of one-pump fiber optical parametric amplifiers. IEEE J Sel Top Quantum Electron. 2004;10:1133-43.

9. Jazayerifar M, Warm S, Elschner R, kroushkov d, Sackey I, Meuer C, et al. Performance evaluation of DWDM communication systems with fiber optical parametric amplifiers. IEEE J Lightwave Technol. 2013;31:1454-62.

10. Mussot A, Kudlinski A, Habert R, Dahman I, Mélin G, Galkovsky L, et al. $20 \mathrm{THz}$-bandwidth continuous-wave fiber optical parametric amplifier operating at $1 \mu \mathrm{m}$ using a dispersion-stabilized photonic crystal fiber. Opt Express. 2012;20: 28906-11.

11. Bobrovs V, Olonkins S, Spolitis S, Porins J, Ivanovs G. Optical fiber and wireless communication. Rastislav Roka, Chapter-10, Evaluation of Parametric and Hybrid Amplifier Applications in WDM Transmission Systems. 2017, IntechOpen, DOI: $10.5772 / 67607$.

12. Jiang $X$, Jiang C. Transmission performance analysis of fiber optical parametric amplifiers for WDM system. Adv Optoelectron. 2009;2009:open access.

13. Zhi Tong Z, Bogris A, Karlsson M, Andrekson PA. Full characterization of the signal and idler noise figure spectra in singlepumped fiber optical parametric amplifiers. Opt Express. 2010;18:2884-93.
14. Golovchenko E, Mamyshev PV, Pilipetskii AN, Dianov EM. Mutual influence of the parametric effects and stimulated Raman scattering in optical fibers. IEEE J Quantum Electron. 1990;26:1815-20.

15. Rottwitt K, Bromage J, Stentz AJ, Leng L, Lines ME, Smith H. Scaling of the Raman gain coefficient: applications to germanosilicate fibers. IEEE J Lightwave Technol. 2003;21:1652-63.

16. Islam MN. Raman amplifiers for telecommuincation. IEEE J Sel Top Qunatum Electron. 2002;8:548-60.

17. Bromage J. Raman amplification for fiber communication systems. IEEE J Lightwave Technol. 2004;22:79-94.

18. Wang SH, Xu L, Wai PKA, Tam HY. Optimization of Ramanassisted fiber optical parametric amplifier gain. IEEE J Lightwave Technol. 2011;29:1172-82.

19. Singh S, Kaler RS. Review on recent developments in hybrid optical amplifier for dense wavelength division multiplexed system. Opt Eng. 2015;54:open access.

20. Redyuk A, Stephens MFC, Doran NJ. Suppression of WDM four-wave mixing crosstalk in fibre optic parametric amplifier using Raman-assisted pumping. Opt Express. 2015;23:27240-9.

21. Wang H, Wang D, Lu C, Cheng TH, Wai PKA. Multiple Raman pump assisted fiber optical parametric amplifiers. IEEE J Lightwave Technol. 2011;29:2601-10.

22. Wang SH, Wai PKA. Gain enhancement in hybrid fiber Raman/ parametric amplifiers. CLEO/QELS: 2010 Laser Science to Photonic Applications, San Jose, CA, 2010:1-2, DOI: 10.1364/CLEO.2010.jTuD56.

23. Ummy MA, Arend MF, Leng L, Madamopoulos N, Dorsinville R. Extending the gain bandwidth of combined Raman-parametric fiber amplifiers using highly nonlinear fiber. IEEE J Lightwave Technol. 2009;27:583-90.

24. Peiris S, Madamopoulos N, Antoniades N, Richards D, Ummy $M A$, Dorsinville R. Engineering an extended gain bandwidth hybrid Raman-optical parametric amplifier for next generation CWDM PON. IEEE J Lightwave Technol. 2014;32:939-47.

25. Stephens MFC, Phillips ID, Rosa P, Harper P, Doran NJ. Improved WDM performance of a fibre optical parametric amplifier using Raman-assisted pumping”. Opt Express. 2015;23:902-11.

26. Gao MJ, Hu W. Dual-pump broadband fiber optical parametric amplifier with a three-section photonic crystal fiber scheme. Proc SPIE Passive Compon Fiber-Based Devices. 2005;5623:300-8.

27. Kaur G, Kaur G, Sharma S. Enhanced gain using Raman FOPA hybrid amplifier for $L$ band $96 \times 100$ Gbps DWDM systems. In: IEEE Conferences Next Generation Computing Technologies. Sept 2015.

28. Kaur G, Kaur G, Sharma S. Multi-section optical parametricRaman hybrid amplifier for Terabit + WDM systems. J Mod Opt. 2016;63:819-25.

29. Boiyo DK, Kuja S, Rotich Kipnoo EK, Waswa D, Muguro KM, Amolo G. Modelling of an optical fibre Raman amplifier. In: Proceedings Conference on Sustainable Research and Innovation. vol. 4, 3rd-4th May 2012.

30. Agrawal GP. Non-linear fiber optics. 4th ed. Cambridge, United States: Academic Press, 2007. 
31. Kidorf H, Rottwitt K, Nissov M, Ma M, Rabarijaona E. Pump interactions in a 100-nm bandwidth Raman amplifier. IEEE Photonics Technol Lett. 1999;11:530-2.

32. Kylemark P, Hedekvist PO, Karlsson M, Torounidis T, Andrekson PA. Noise characteristics in fiber optical parametric amplifiers. IEEE J Lightwave Technol. 2004;22:409-16.

33. Singh S, Kaler RS. Performance optimization of EDFA-raman hybrid optical amplifier using genetic algorithm. Opt Laser Technol. 2015;68:89-95.

34. Lavery D, Liu S, Jeong Y, Nilsson J, Petropoulos P, Bayvel P, et al. Realizing High Sensitivity at 40Gbit/s and 100Gbit/. IEEE/
OSA Opt Fiber Commun Tech Dig. 2012. DOI: 10.1364/OFC.2012. OW3H.5.

35. Lach E, Idler W. Modulation formats for $100 \mathrm{~g}$ and beyond. J Opt Fiber Technol. 2011;17:377-86.

36. Malik R, Kumpera A, Karlsson M, Andrekson PA. Demonstration of ultra wideband phase-sensitive fiber optical parametric amplifier. IEEE Photonics Technol Lett. 2016;28:175-7.

37. Marhic ME, Andrekson PA, Petropoulos P, Radic S, Peucheret C, Jazayerifar M. Fiber optical parametric amplifiers in optical communication systems. Laser Photonics Rev. 2015;9:50-74. 